Ann. Zootech., I980, 29 (3), 299-304.

\title{
Activités alimentaires nocturnes du cheval au pâturage
}

\author{
M. DOREAU, W. MARTIN-ROSSET ${ }^{(1)}$ et D. PETIT (1) \\ Laboratoire de la Production Laitière \\ (1) Laboratoire de la Production de Viande \\ Centre de Recherches de Clermont-Ferrand, I.N.R.A. \\ Theix, 63IIo Beaumont (France)
}

\begin{abstract}
Résumé
Les activités alimentaires nocturnes du cheval au pâturage ont été étuđiées sur 4 pouliches munies d'enregistreurs automatiques, pendant deux périodes (juin et août).

Les chevaux consacrent à l'ingestion $36 \mathrm{p}$. roo de la nuit; la durée d'ingestion nocturne représente en moyenne 23 p. Ioo de la durée d'ingestion totale, à laquelle elle est liée linéairement. Cependant, la prédiction de la durée d'ingestion totale à partir de la durée d'ingestion diurne est obtenue avec une erreur résiduelle de $58 \mathrm{mn}$. La durée d'ingestion nocturne semble dépendre de la période et de l'animal, mais ces différences n'ont pas été significatives, en raison d'importantes variations d'un jour à l'autre.

L'examen des activités heure par heure a permis de mettre en évidence d'une part l'influence du lever et du coucher du soleil sur les activités alimentaires, d'autre part un rythme périodique dont l'origine est discutée.
\end{abstract}

\section{Introduction}

Les activités alimentaires du cheval au pâturage ont déjà été étudiées pendant la journée (Rogalski, I970, I975; Martin-Rosse't, DorEaU, Cloix, I978). Les activités nocturnes sont en revanche peu connues : selon KUROK1 et 1KEDA (I974) puis ArNold et DudzInsku (I978), la durée d'ingestion nocturne des chevaux apparaît non négligeable. Par ailleurs, chez le cheval ou le poney à l'auge, recevant des fourrages ad libitum en deux distributions, matin et soir, elle représente entre 24 et 35 p. Ioo de la durée d'ingestion totale (RUCKEBUSCH, VIGROUX, CANDAU, I976; DOREaU, I978).

Aussi avons-nous voulu préciser l'importance de l'ingestion nocturne du cheval au pâturage et ses variations éventuelles avec la saison, la durée d'ingestion totale et entre animaux. Pour cela, nous avons procédé à des enregistrements en continu des mouvements de la mâchoire sur un afpareil portatif. 


\section{Matériel et méthodes}

Nous avons utilisé 4 pouliches de 15 mois, de race Bretonne ou Comtoise. Eilles exuloitaient en rotation, au sein d'un troupeau de ro pouliches, une prairie naturelle située à I $400 \mathrm{~m}$ d'altitude, riche en espèces ligneuses ( $25 \mathrm{p}$. Ioo de la biomasse) décrite par ailleurs (MARTIN-ROSSET, LOISEAU, I979). Le chargement était de $365 \mathrm{~kg}$ de poids vif par ha.

Deux séries de mesure (de 6 et 5 jours) situées au milieu des deuxpremiers cycles d'exploitation de la même parcel1e, se sont déroulées en fin juin et début août I978. La durée d'ingestion était mesurée grâce à l'appareil décrit par RUCKEBUSCH, BUENo et LATOUR (I973), modifié par BÉCHET (I978) : les mouvements de la mâchoire sont transmis par voie pneumatique à un enregistreur effectuant une transcription graphique; ce!ui-ci est maintenu dans une sacoche accrochée au licol des animaux. Le changement du disque d'enregistrement s'effectuait entre I5 $\mathrm{h}$ et $\mathrm{I} 7 \mathrm{~h}$. Les manipulations des animaux ont été plus longues en août qu'en juin; aussi la durée totale de mesure journalière a-t-elle été de $23 \mathrm{~h}$ 4I $\mathrm{mn}$ en première période et $23 \mathrm{~h}$ II mn en deuxième, soit 98,7 et 96,5 p. Ioo de la durée d'une journée.

Au total, 32 enregistrements journaliers ont été obtenus lors de journées sans perturbations climatiques importantes (vent fort, pluie). L'éclairement nocturne dû à la lune était vlus élevé en première période (en moyenne 2 jours après la pleine lune) qu'en deuxième (en moyenne 4 jours après la nouvelle lune).

Les heures du lever et du coucher du soleil étaient 4 h et $20 \mathrm{~h}$ G.M.T. en juin, $4 \mathrm{~h} 30$ et I 9 h 30 G.M.T. en aô̂t. Nous avons appelé "nuit "la phase comprise entre $20 \mathrm{~h}$ et $4 \mathrm{~h}$ G.M.T.

\section{Résultats}

La durée d'ingestion totale journalière moyenne est de $\mathrm{I} 2 \mathrm{~h} 2 \mathrm{I} \mathrm{mn}$. Elle ne diffère pas significativement entre périodes : une analyse de covariance a été effectuée afin de tenir compte de la différence de $30 \mathrm{mn}$ entre les durées de mesure des deux périodes. En revanche, la variation d'un jour à l'autre est élevée.

L'activité alimentaire occupe en moyenne plus de $35 \mathrm{p}$. Ioo de la phase nocturne. Cette proportion a été plus importante en période $\mathbf{I}$ qu'en période 2 . L'ingestion alimentaire nocturne est un phénomène général, puisque elle constitue plus de $18 \mathrm{p}$. Ioo de la phase nocturne dans 30 observations sur 32 .

La durée d'ingestion nocturne représente $23 \mathrm{p}$. Ioo de la durée d'ingestion. totale. Cette proportion est plus élevée en période I qu'en période 2 , elle est de Ig p. Ioo environ de l'ingestion totale pour deux animaux et $25 \mathrm{p}$. roo pour les deux autres. Mais ces différences ne sont pas significatives : une analyse de covariance a été effectuée pour tenir compte đe la différence de durée d'ingestion totale entre les animaux. La durée d'ingestion nocturne apparaît liée linéairement à la durée totale d'ingestion (fig. I) : $r=0,73 ; n=32$. Un ajustement non linéaire n'améliore pas le coefficient de corrélation. Il apparaît que même lorsque la durée d'ingestion totale est faible, le cheval maintient une activité alimentaire nocturne. 


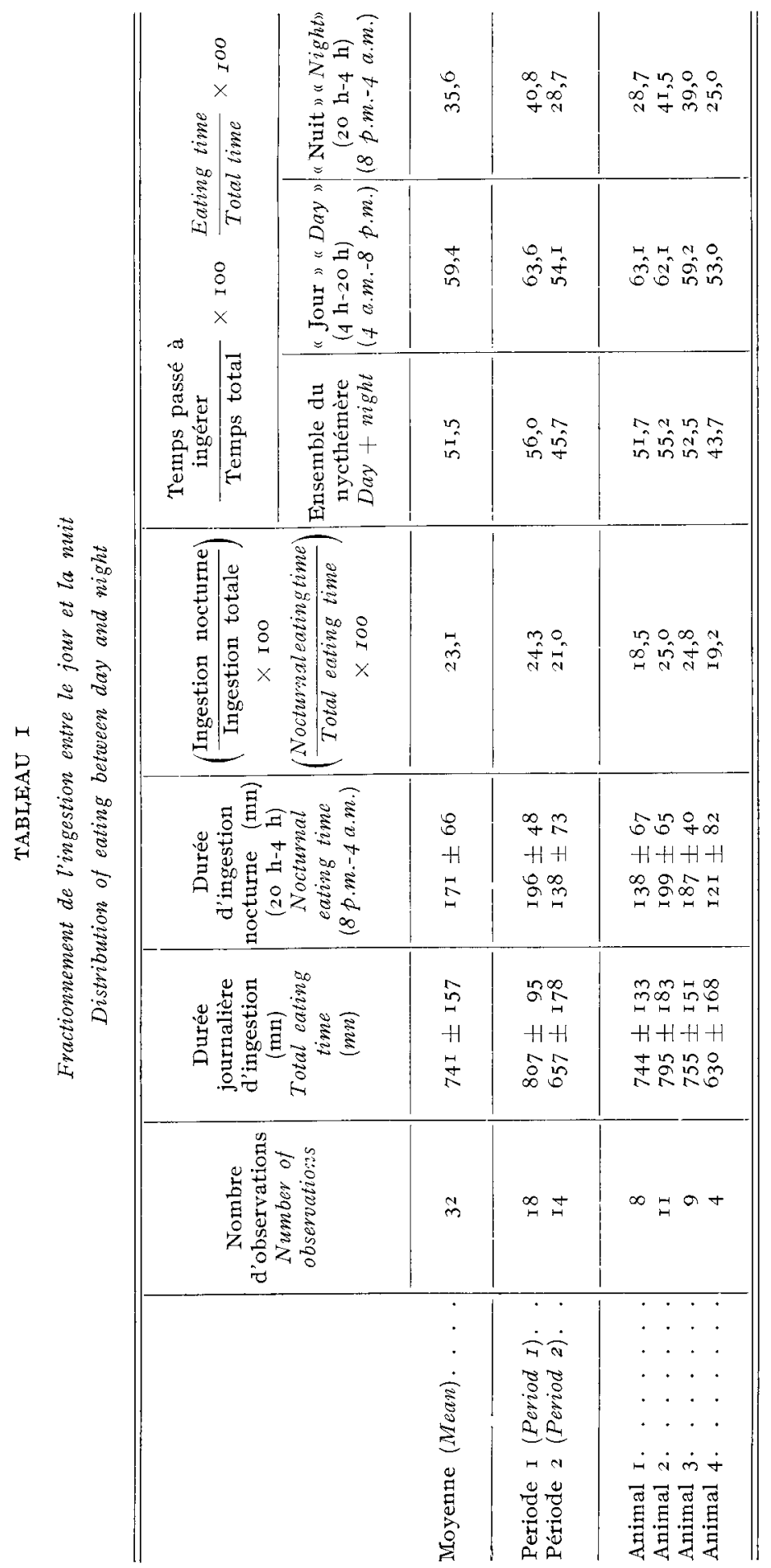




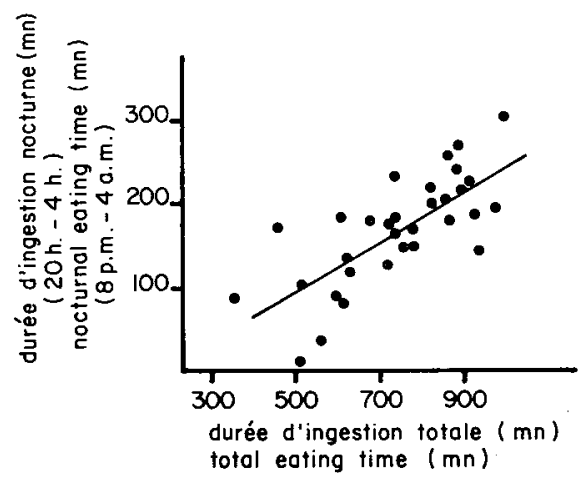

FIG. I. - Relation entre la durée d'ingestion nocturne et la durée d'ingestion totale. Relation between nocturnal and total eating time.

La relation de prédiction de la durée d'ingestion totale ( $y$ en $\mathrm{mn}$ ) en fonction de la durée d'ingestion diurne ( $x$ en $\mathrm{mn}$ ) s'exprime : $y=\mathrm{I}, \mathrm{I} 9 x+54$. Le coefficient de corrélation est élevé $(r=0,93)$, mais l'erreur résiduelle est importante $(\mathrm{S} y x=58 \mathrm{mn})$.

La figure 2 montre la répartition nycthémérale de l'ingestion. Deux faits s'en dégagent. En premier lieu, le lever du jour et la tombée de la nuit ont un effet prépondérant. Le premier provoque un accroissement important du temps passé à ingérer et la seconde une diminution encore plus brutale. D'ailleurs, le décalage des horaires de lever et de coucher du soleil entre les deux périodes se retrouve au niveau des activités alimentaires, particulièrement lors du coucher du soleil. En second lieu, l'ingestion varie de manière sinusoïdale avec une périodicité voisine de 6 heures. Ceci correspond pour la phase diurne à trois maxima d'ingestion (4-6 h, II-I 3 h, I 7-20 h G.M.T.) et à deux minima (8-Io h et I3-I4 h G.M.T.) et pour la phase nocturne à un maximum (22-o h G.M.T.) et deux minima (2I-22 h et I-2 h G.M.T.).

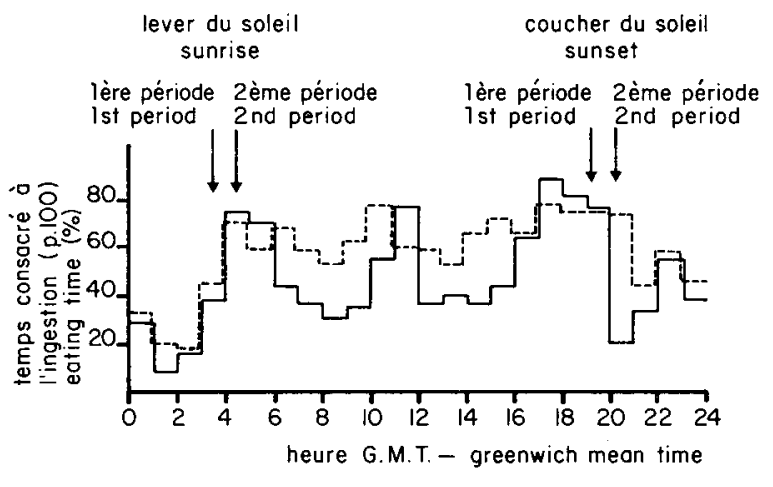

FIG. 2. - Répartition nycthémérale de l'ingestion au paturage. Circadian pattern of eating at pasture. 


\section{Discussion}

Ces résultats mettent en évidence l'importance du pâturage nocturne chez le cheval. Ils semblent indiquer que les activités alimentaires nocturnes ne sont pas uniquement pour l'animal un moyen de prolonger sa durée d'ingestion. En effet, même lorsque la durée d'ingestion totale est courte, le cheval s'alimente la nuit; par ailleurs, la durée d'ingestion nocturne augmente linéairement avec la durée d'ingestion totale. RUCKEBUSCH, VIGROUX et CANDAU (I976) avaient effectué la même constatation : des poneys à 1'auge alimentés ad libitum et consommant soit des fourrages soit des aliments concentrés, ce qui conduit à des durées d'ingestion très différentes, répartissent de manière similaire leur ingestion entre le jour et la nuit. Il en est de même pour des chevaux à l'auge consommant ad libitum le même régime à des niveaux d'ingestion très différents; toutefois, l'ingestion nocturne est plus faible chez les animaux rationnés (DOREAU, I 978). Le faible nombre d'observations réalisées dans cet essai permet difficilement de mettre en évidence des facteurs de variation de l'ingestion nocturne tels que l'effet individuel ou l'influence de la clarté nocturne.

L'influence du lever du jour et de la tombée de la nuit sur le déclenchement et l'arrêt des activités alimentaires devrait être précisée par des observations au printemps et à l'automne; ces premiers résultats sont néanmoins comparables à ceux obtenus sur bovins (RUCKFBUSCH, BUENo, I978) ou ovins (THERIEZ, BÉCHET, Molenat, I979) au pâturage. Les pointes régulières d'activités alimentaires, environ toutes les six heures, peuvent être attribuées à un rythme biologique. Chez le cheval à l'auge alimenté ad libitum et répartissant son ingestion en fonction des deux distributions journalières d'aliments, on observe également un maximum et deux minima d'activité nocturne (Dorkau, I978). Cependant, dans cet essai, la périodicité peut également s'expliquer par un "effet troupeau ": les équidés regroupent leurs activités alimentaires en cycles au niveau du troupeau (KI,INGEL, I967), donc probablement en fonction du rythme d'activité de l'animal dominant. Elle peut également dépendre de la quantité et de la qualité des apports alimentaires, ce qui expliquerait partiellement ses variations avec la saison (MARTINRosset, Doreau, Clotx, r978). Dans ce cas, la périodicité serait déterminée par des informations d'origine digestive ou métabolique.

La relation entre les durées d'ingestion diurne et totale est suffisamment étroite pour que les résultats établis grâce à des observations diurnes (effet de la saison, du climat, des caractéristiques des animaux sur la durée d'ingestion) ne soient pas remis en question par des variations des activités nocturnes. Cependant, la prédiction individuelle des durées d'ingestion totales à partir des durées diurnes est rendue aléatoire en raison d'une erreur résiduelle importante.

Accepté pour publication en juin 1980.

\section{Summary}

\section{Nocturnal feeding activities of horses at pasture}

Feeding activities of horses at pasture have been studied by automatic recording of jaw movements (BECHA 1 , 1978 ) on 4 fillies during 2 periods (june and august).

Main results are reported on table $\mathrm{I}$. Horses spend $36 \mathrm{p}$. Ioo time on eating. Mean nocturnal time is $23 \mathrm{p}$. I oo of total eating time: both are linearly linked (fig. I). However, predicting 
total eating time from diumal eating time is made with a residual error of 58 mn. Nocturnal eating time depends on the period and the animal, but these differences are not significant, day to day variations being important.

Hourly feeding pattern (fig. 2) shows: r) the effect of sunrise and sunset on feeding activities; 2) a periodic rhythm, whose origin is discussed.

\section{Références bibliographiques}

ARNor,D G. W., Dudzinski M. L., I978. Ethology of free-ranging domestic animals. I vol., I 98 p. Ed. Eilsevier, Ansterdam.

BECHET G., I $97^{8}$. Enregistrement des activités alimentaires et méryciques des ovins au pâturage. Ann. Zootech., 27, I07-1 I3.

Doreau M., I978. Comportement alimentaire du cheval à l'écurie. Ann. Zootech., 27, 29 I-302.

KLINGEL H., I967. Soziale Organisation und Verhalten freilebender Steppenzebras. $Z$. Tierpsychol., 24, 580-624.

KUROKI M., IKEDA M., I974. Studies on the Misaki horse. II. - On the grazing behaviors. Bull. Fac. Agric. Miyazaki Univ., Miyazak, Japan, 21, 315-322.

Martin-Rosset W., Doreau M., Cloix J., I978, Étude des activités d'un troupeau de poulinières de trait et de leurs poulains au pâturage. Ann. Zootech., 27, 33-45.

Martin-Rosset W., LoIsEaU P., I979. Récupération des pâturages dégradés par les chevaux. C.R. $5^{\mathrm{e}}$ Journée d'étude CEREOPA, 49-66. Éd. CEREOPA Paris.

RoGalSki M., I970. Comportement des chevaux au pâturage (en polonais). Kon Polski, 5 (4), 26-27.

RoGaISKI M., 1975. Effet des conditions climatiques et du mode de conduite sur le comportement des chevaux au pâturage (en polonais). Roczn. Nauk. Roln., B 97 (r), 7-I6.

RUCKEBUSCh Y., BuENo L., LATOUR A., 1973. Recherches sur le comportement alimentaire des ruminants. Io. Activités alimentaires des bovins au pâturage. Rev. Med. Vet., 124, 685-69I.

Ruckebusch Y., Vigroux P., Candau M., i976. Analyse du comportement alimentaire chez les Équidés. C.R. $2^{\mathrm{e}}$ Journée d'étude CEREOPA, 69-72, Ed. CEREOPA, Paris.

RUCKEBUSCH Y., BUENO L., 1978. An analysis of ingestive behaviour and activity of cattle utlder field conditions. Appl. Anim. Ethol., 4, 30I-313.

Theriez M., Bechet G., Molena'T G., i979. Ingestive behaviour of sheep at pasture. Proc. 3oth Ann. Meet. Europ. Assoc. Anim. Prod., Harrogate, G. B., 6 p. 